\title{
Pigmented hepatocellular adenomas have a high risk of atypia and malignancy
}

\author{
Taofic Mounajjed, Saba Yasir, Patrice A Aleff and Michael S Torbenson \\ Division of Anatomic Pathology, Department of Laboratory Medicine and Pathology, Mayo Clinic, Rochester, \\ $M N, U S A$
}

\begin{abstract}
Pigment deposition is occasionally seen in hepatocellular adenomas. Several reports suggest that pigmented hepatocellular adenomas have increased risk of malignancy, but these tumors remain incompletely understood. To determine the frequency of pigment deposition, we evaluated and classified 109 well-differentiated hepatocellular neoplasms that were originally diagnosed or submitted in consultation as hepatocellular adenomas and found $27(25 \%)$ pigmented tumors. All were negative on iron stain and in three cases electron microscopy confirmed the pigment was lipofuscin. The lipofuscin intensely stained with glypican-3 in most cases $(89 \%)$. Of the 27 pigmented tumors, 11 cases $(41 \%)$ were classified as hepatocellular adenomas, 7 cases $(27 \%)$ were classified as atypical hepatocellular adenomas/hepatocellular neoplasms of uncertain malignant potential, and 9 cases $(33 \%)$ were reclassified as well-differentiated hepatocellular carcinomas. Four (of 9 ) hepatocellular carcinomas arose in pigmented hepatocellular adenomas, giving a rate of malignant transformation in pigmented hepatocellular adenomas of $27 \%$. Of the total 27 pigmented tumors, $78 \%$ were in women and $22 \%$ in men, but interestingly only men had tumors classified as hepatocellular carcinoma or hepatocellular neoplasm of uncertain malignant potential. Also of note, a total of 10 individuals $(37 \%)$ had multiple hepatocellular neoplasms but in 9 of these cases the other adenomas were non-pigmented. Importantly, in cases with multiple hepatocellular neoplasms, only the pigmented hepatocellular neoplasms had atypia or malignancy. Genotype-phenotype classification of the pigmented tumors showed different subtypes: HNF1a inactivated (48\%), $\beta$-catenin activated $(26 \%)$, inflammatory $(15 \%)$, concurrently $\beta$-catenin activated and inflammatory in 1 hepatocellular adenoma, concurrently HNF-1 $\alpha$ inactivated and $\beta$-catenin activated in 1 hepatocellular adenoma, and unclassified in 1 hepatocellular carcinoma. In conclusion, hepatocellular adenomas with lipofuscin pigment are a heterogeneous group of adenomas, with HNF-1a inactivation being the commonest genotype. They have an increased risk of atypia and malignancy, especially in males.
\end{abstract}

Modern Pathology (2015) 28, 1265-1274; doi:10.1038/modpathol.2015.83; published online 24 July 2015

Hepatocellular adenomas are rare; they affect 3-4/100,000 individuals in North America and Europe. ${ }^{1}$ They represent a multifactorial disease in which gender (female), hormones (estrogen and androgens), and genetic predisposition have an important role. ${ }^{2-4}$ The genotype-phenotype classification of hepatocellular adenomas, ${ }^{4-8}$ recently recognized by the world health organization, ${ }^{9}$ has provided valuable insights into the pathogenesis and clinicopathological features of hepatocellular adenomas. Certain variants of hepatocellular adenomas, however, are still not fully characterized.

Correspondence: Dr T Mounajjed, MD, Division of Anatomic Pathology, Department of Laboratory Medicine and Pathology, Mayo Clinic, Hilton 11, 200 1st Street SW, Rochester, MN 55905, USA.

E-mail: Mounajjed.Taofic@mayo.edu

Received 1 April 2015; revised 22 May 2015; accepted 26 May 2015; published online 24 July 2015
For instance, although pigment deposition in hepatocellular adenomas has been reported in rare cases (often referred to as pigmented hepatocellular adenoma $)^{10-15}$ and even rarer instances of hepatocellular carcinoma, ${ }^{16,17}$ the overall pathological features of pigmented hepatocellular neoplasms have not been well characterized. A total of eight cases of pigmented hepatocellular adenomas have been reported in the literature, ${ }^{10-15}$ but the phenotype of only two cases has been characterized. ${ }^{10,15}$ Furthermore, the malignant potential of these lesions is still unclear; as some studies have suggested that these lesions are essentially benign, ${ }^{12,13}$ whereas others have described atypia and malignant transformation. ${ }^{10,15}$ Finally, although believed to be rare, the exact frequency of pigment deposition in hepatocellular adenomas is unknown.

Hence, because these tumors are not well characterized, we reviewed 109 well-differentiated 
hepatocellular neoplasms to identify and further characterize pigmented tumors. The purpose of this study is to describe the overall frequency, phenotype, and risk of malignancy associated with pigment deposition in well-differentiated hepatocellular neoplasms.

\section{Materials and methods}

A total of 109 well-differentiated hepatocellular neoplasms (73 resections and 36 biopsies) originally diagnosed or submitted for consultation as hepatocellular adenomas or atypical hepatocellular adenomas (between 1990 and 2014), were retrospectively reviewed for intracytoplasmic pigment deposition within hepatocytes. We initially set out to include only cases with very prominent pigment deposition comparable to that described in the literature, but as we evaluated all lesions, we noticed that there is a spectrum of pigment deposition in hepatocellular neoplasia, with variability both in the amount and distribution of pigmentation. Although we aimed to characterize a distinct morphological group, we also realized that we had to account for this variability. Hence, we decided to include not only lesions with very prominent pigmentation, but any hepatocellular neoplasm with 'easily detected' pigment; any tumor with pigment identifiable at $100 \times$ magnification was considered a pigmented neoplasm for the purposes of this study. Pigment deposition was then semiquantitatively scored as follows: 0 : no pigment identifiable at $100 \times$ magnification, 1: pigment present focally (minority of $100 \times$ fields), 2: pigment identified easily at $100 \times$ magnification (majority of $100 \times$ fields), 3: pigment easily identified at $40 \times$ magnification. On the basis of these criteria, 27 pigmented tumors $(25 \%)$ were identified (24 resections and 3 biopsies).

All lesions were subjected to clinical record review and histopathological examination by three pathologists (TM, SY, and MST). On the basis of the histological features and blinded to all clinical data, the pigmented hepatocellular neoplasms were classified as hepatocellular adenomas, hepatocellular neoplasms of uncertain malignant potential, or hepatocellular carcinomas. A diagnosis of hepatocellular carcinoma was made if the tumor showed definite loss of reticulin, which was typically accompanied by significant cytological atypia, increased cell proliferation, and architectural atypia. Architectural atypia included thickened trabeculae, pseudoacini, or nodule within nodule growth. In contrast, hepatocellular adenomas had no cytological or architectural atypia, no increased proliferation, and no reticulin reduction. Tumors that showed focal atypical features, insufficient to make a diagnosis of hepatocellular carcinoma, but unusual for typical hepatocellular adenoma were labeled 'hepatocellular neoplasms of uncertain malignant potential'. To make this diagnosis, we followed the criteria proposed by Bedossa et al; these criteria include focal cytological atypia (small cell change or nuclear atypia) involving $<5 \%$ of the tumor or focal architectural atypia (pseudoglandular forms) involving $<5 \%$ of the tumor or focal reticulin loss. ${ }^{18}$

Histological examination also included evaluation of the background liver for prominence of lipofuscin pigment in cases that contained sufficient nonneoplastic liver for evaluation (19 cases). Lipofuscin deposition in the background liver was semiquantitatively graded (0-3) using the same criteria utilized for pigment scoring in the neoplasms.

A control group, consisting of 19 age and gender matched non-pigmented hepatocellular adenomas, was also subject to histological evaluation including evaluation of lipofuscin pigment in the background liver. Student's $t$-test was used to evaluate for difference in lipofuscin deposition between the pigmented hepatocellular neoplasm group and the control group; a $P$-value $<0.05$ was considered statistically significant.

Special stains for iron and reticulin were performed on all pigmented tumors. All pigmented tumors were also subject to immunohistochemical evaluation with liver fatty acid-binding protein, $\beta$-catenin, glutamine synthetase, C-reactive protein, serum amyloid-A, glypican-3, and ki-67. Immunohistochemistry was performed using steam antigen retrieval and established methodologies with antibodies for serum amyloid-A (1:2000, clone mc1; Dako, CA, USA), C-reactive protein (1:2000, clone Y284; Abcam, MA, USA), glutamine synthetase (1:2000, clone GS-6; Millipore, MA, USA), $\beta$-catenin (predilute, clone 14; Ventana, AZ, USA), liver fatty acid-binding protein (1:50, rabbit polyclonal; Abcam, MA, USA), glypian-3 (predilute, mouse, monoclonal, clone 1G12, Ventana, CA, USA), and Ki-67 (1:20, clone MIB1; Dako). The immunohistochemical profile was utilized to classify tumors according to the hepatocellular adenoma genotype-phenotype classification, as showing $\beta$ catenin activation, HNF-1 $\alpha$ inactivation, or inflammatory phenotype.

A subset of three cases was also selected based on their pigment staining characteristics for evaluation by electron microscopy. Ultrastructural analysis was performed using formalin-fixed, paraffin-embedded tissue, which was deparaffinized, routinely processed, Epon-embedded, and stained with uranyl acetate and lead citrate. Thin sections were examined on a Tecnai 12 model transmission electron microscope (FEI Corp, Eindhoven Netherlands).

\section{Results}

Table 1 and Table 2 summarize the histological and immunohistochemical features of pigmented hepatocellular tumors. Of the 27 pigmented tumors, 11 cases $(41 \%)$ were classified as hepatocellular adenoma, $7(26 \%)$ were classified as hepatocellular neoplasm of uncertain malignant potential, and 9 
Table 1 Summary of histological and immunohistochemical features of pigmented hepatocellular neoplasms

\begin{tabular}{|c|c|c|c|}
\hline \multirow[b]{2}{*}{ Classification } & \multicolumn{3}{|c|}{ Pigmented hepatocellular neoplasms } \\
\hline & Hepatocellular adenoma (11) & $\begin{array}{l}\text { Hepatocellular neoplasm of } \\
\text { uncertain malignant potential ( } 7)\end{array}$ & $\begin{array}{l}\text { Hepatocellular } \\
\text { carcinoma (9) }\end{array}$ \\
\hline Average age & $39 \pm 11$ years & $45 \pm 17$ years & $32 \pm 18$ years \\
\hline Gender & 11 Female & $\begin{array}{l}5 \text { Female } \\
2 \text { Male }\end{array}$ & $\begin{array}{l}5 \text { Female } \\
4 \text { Male }\end{array}$ \\
\hline Average size & $7.8 \pm 4.3 \mathrm{~cm}$ & $6 \pm 4.6 \mathrm{~cm}$ & $9.9 \pm 7.1 \mathrm{~cm}$ \\
\hline Multiple lesions & $6(55 \%)$ & $3(43 \%)$ & $1(11 \%)$ \\
\hline Pigment score (number of lesions) & $\begin{array}{l}1(6) \\
2(2) \\
3(3)\end{array}$ & $\begin{array}{l}1(2) \\
2(4) \\
3(1)\end{array}$ & $\begin{array}{l}1(4) \\
2(2) \\
3(3)\end{array}$ \\
\hline Cytological atypia & None & 2 of $7(29 \%)$ & 6 of $9(67 \%)$ \\
\hline Architectural atypia & None & 5 of $7(71 \%)$ & 7 of $9(78 \%)$ \\
\hline Reticulin & Intact (11 of $11,100 \%)$ & $\begin{array}{c}6(86 \%) \text { intact } \\
1(14 \%) \text { focally disrupted }\end{array}$ & $\begin{array}{c}4 \text { (44\%) focally disrupted } \\
5(56 \%) \text { diffusely } \\
\text { disrupted }\end{array}$ \\
\hline Ki-67 & $<1 \%(11$ of $11,100 \%)$ & $\begin{array}{l}<1 \% \text { in } 6(86 \%) \\
2 \% \text { in } 1(14 \%)\end{array}$ & $\begin{array}{l}<1 \% \text { in } 8(89 \%) \\
7 \% \text { in } 1(11 \%)\end{array}$ \\
\hline Immunohistochemical profile & $\begin{array}{c}\text { HNF- } 1 \alpha \text { inactivated }(8,73 \%) \\
\text { Inflammatory }(1,9 \%) \\
\text { HNF- } 1 \alpha \text { inactivated and } \\
\beta \text {-catenin activated }(1,9 \%) \\
\text { Inflammatory and } \beta \text {-catenin } \\
\text { activated }(1,9 \%)\end{array}$ & $\begin{array}{c}\text { HNF- } 1 \alpha \text { inactivated }(4,57 \%) \\
\text { Inflammatory }(2,29 \%) \\
\beta \text {-catenin activated }(1,14 \%)\end{array}$ & $\begin{array}{c}\text { HNF- } 1 \alpha \text { inactivated } \\
(1,11 \%) \\
\text { Inflammatory }(1,11 \%) \\
\beta \text {-catenin activated } \\
(6,67 \%) \\
\text { Unclassified }(1,11 \%)\end{array}$ \\
\hline
\end{tabular}

(33\%) were classified as hepatocellular carcinoma (Figure 1). None of the hepatocellular adenomas had cytological or architectural atypia. In contrast, cytological or architectural atypia were encountered in hepatocellular neoplasms of uncertain malignant potential (29\% and $71 \%$, respectively) and hepatocellular carcinomas (67\% and $78 \%$, respectively). In the hepatocellular neoplasm of uncertain malignant potential group, neither cytological nor architectural atypia increased with age (Table 2), and males had more architectural atypia ( 2 of 2 in males compared with 3 of 5 in females) and less cytological atypia (0 of 2 in males compared with 2 of 5 in females). The nine cases classified as hepatocellular carcinoma were all well differentiated. Four hepatocellular carcinomas arose as distinct nodules in hepatocellular adenomas, with the adenoma recognizable at the periphery of the carcinoma (Figure 2), giving a 'nodule in nodule' appearance.

Most individuals (21 of 27: 78\%) were women (hepatocellular adenomas: 11 of 11; hepatocellular neoplasms of uncertain malignant potential: 5 of 7; hepatocellular carcinoma: 5 of 9). None of the pigmented tumors in males were classified as hepatocellular adenoma, with 4 of 6 classified as hepatocellular carcinoma and 2 as hepatocellular neoplasms of uncertain malignant potential.

Individuals ranged in age between 18 and 60 years (average age: 38 years for all cases; 39 years for hepatocellular adenoma, 45 years for hepatocellular neoplasm of uncertain malignant potential, and 32 years for hepatocellular carcinoma). Tumors ranged in size between 1.5 and $21 \mathrm{~cm}$ (average size: $8.1 \mathrm{~cm}$ for all cases, $7.8 \mathrm{~cm}$ for hepatocellular adenoma,
$6 \mathrm{~cm}$ for hepatocellular neoplasm of uncertain malignant potential, and $9.9 \mathrm{~cm}$ for hepatocellular carcinoma). None of the patients had significant background liver fibrosis/cirrhosis. Only one patient with hepatic adenomatosis had glycogen storage disease, but none of the other patients had known background liver disease.

Ten (37\%) individuals had multiple lesions, ranging in number between 2 and $>50$. In this subset of cases, the pigmented tumors were classified as hepatocellular adenomas (6 cases), hepatocellular neoplasms of uncertain malignant potential (3 cases), and hepatocellular carcinoma (1 case). In 9 of the 10 individuals with multiple lesions, the other lesions were not pigmented and were all adenomas. Of note, in all four cases with hepatocellular neoplasms of uncertain malignant potential or carcinoma, it was only the pigmented lesions that were atypical or malignant. The non-pigmented lesions showed no atypia and no carcinoma. The only case of multiple pigmented lesions featured two pigmented hepatocellular adenomas (pigment score of 1 and 2).

Fatty change was present in 20 of the 27 pigmented tumors (74\%); overall, fatty change was seen in 9 of 11 hepatocellular adenomas, 6 of 7 hepatocellular neoplasms of uncertain malignant potential, and 5 of 9 hepatocellular carcinomas, and was prominent (exceeding 67\%) in 4 hepatocellular adenomas and 1 hepatocellular neoplasm of uncertain malignant potential.

The pigment was azonal in 21 cases, had a perivascular distribution in 4 cases ( 2 hepatocellular carcinomas and 2 hepatocellular adenomas), and had pericanalicular localization in 2 hepatocellular 
Table 2 Clinicopathological features of all pigmented hepatocellular neoplasms

\begin{tabular}{|c|c|c|c|c|c|c|c|c|c|}
\hline Case & $\begin{array}{l}\text { Classification of } \\
\text { hepatocellular neoplasm }\end{array}$ & $\begin{array}{l}\text { Age, } \\
\text { gender }\end{array}$ & $\begin{array}{c}\text { Number of lesions in } \\
\text { patient }\end{array}$ & Size $(\mathrm{cm})^{\mathrm{a}}$ & Cytological atypia & Architectual atypia & Reticulin & $\begin{array}{l}\text { Pigment } \\
\text { score }^{\mathrm{b}}\end{array}$ & Phenotype \\
\hline 1 & Adenoma & $34, \mathrm{~F}$ & 1 & 11 & No & No & Intact & 2 & HNF1- $\alpha$ inactivated \\
\hline 2 & Adenoma & $35, \mathrm{~F}$ & 1 & 5.2 & No & No & Intact & 1 & $\begin{array}{l}\text { Inflammatory } \beta \text {-catenin } \\
\text { activated }\end{array}$ \\
\hline 3 & Adenoma & $24, \mathrm{~F}$ & 1 & 11.5 & No & No & $\begin{array}{l}\text { Intact (focal fat } \\
\text { associated reduction) }\end{array}$ & 3 & HNF1- $\alpha$ inactivated \\
\hline 4 & Adenoma & $58, \mathrm{~F}$ & 1 & 12 & No & No & Intact & 3 & HNF1- $\alpha$ inactivated \\
\hline 5 & Adenoma & $35, \mathrm{~F}$ & 1 & $\begin{array}{l}\text { Unavailable, biopsy } \\
\text { specimen }\end{array}$ & No & No & Intact & 2 & $\begin{array}{l}\text { HNF1- } \alpha \text { inactivated }+\beta- \\
\text { catenin activated }\end{array}$ \\
\hline 6 & Adenoma & $36, \mathrm{~F}$ & $>50$ & 4.5 & No & No & $\begin{array}{l}\text { Intact (focal fat } \\
\text { associated reduction) }\end{array}$ & 1 & HNF1- $\alpha$ inactivated \\
\hline 7 & Adenoma & $40, \mathrm{~F}$ & 2 & 7.7 & No & No & Intact & 1 & Inflammatory \\
\hline 8 & Adenoma & $57, \mathrm{~F}$ & 2 & 2.9 & No & No & Intact & 1 & HNF1 $-\alpha$ inactivated \\
\hline 9 & Adenoma & $47, \mathrm{~F}$ & 2 & 6 & No & No & Intact & 3 & HNF1- $\alpha$ inactivated \\
\hline 10 & Adenoma & $27, \mathrm{~F}$ & 2 & 15 & No & No & $\begin{array}{l}\text { Intact (focal fat } \\
\text { associated reduction) }\end{array}$ & 1 & HNF1- $\alpha$ inactivated \\
\hline 11 & Adenoma & $31, \mathrm{~F}$ & 2 & 2.4 & No & No & Intact & 1 & HNF1- $\alpha$ inactivated \\
\hline 12 & $\begin{array}{l}\text { Carcinoma arising in } \\
\text { adenoma }\end{array}$ & $34, \mathrm{~F}$ & 1 & 10 & Yes & Yes & Patchy loss & 2 & Unclassified \\
\hline 13 & $\begin{array}{l}\text { Carcinoma arising in } \\
\text { adenoma }\end{array}$ & $55, \mathrm{~F}$ & 1 & 2.4 & Yes & Yes & Loss & 1 & $\beta$-catenin activated \\
\hline 14 & $\begin{array}{l}\text { Carcinoma arising in } \\
\text { adenoma }\end{array}$ & $18, \mathrm{~F}$ & 1 & 1.5 & No & Yes & Patchy loss & 1 & HNF1- $\alpha$ inactivated \\
\hline 15 & $\begin{array}{l}\text { Carcinoma arising in } \\
\text { adenoma }\end{array}$ & $20, \mathrm{M}$ & 1 & 10 & Yes & No & Loss & 1 & Inflammatory \\
\hline 16 & Carcinoma & $37, \mathrm{M}$ & $\begin{array}{l}4 \text { (including } 3 \text { non- } \\
\text { pigmented adenomas) }\end{array}$ & 3.7 & No & Yes & Patchy loss & 1 & $\beta$-catenin activated \\
\hline 17 & Carcinoma & $42, \mathrm{~F}$ & 1 & 21 & Yes & Yes & Patchy loss & 3 & $\beta$-catenin activated \\
\hline 18 & Carcinoma & $5, \mathrm{M}$ & 1 & 15 & Yes & Yes & Patchy loss & 2 & $\beta$-catenin activated \\
\hline 19 & Carcinoma & $54, \mathrm{~F}$ & 1 & 6.4 & Yes & No & Loss & 3 & $\beta$-catenin activated \\
\hline 20 & Carcinoma & $24, \mathrm{M}$ & 1 & 19 & No & Yes & Loss & 3 & $\beta$-catenin activated \\
\hline 21 & $\begin{array}{l}\text { Uncertain malignant } \\
\text { potential }\end{array}$ & $29, \mathrm{~F}$ & 1 & 8.5 & Yes & No & Intact & 2 & Inflammatory \\
\hline 22 & $\begin{array}{l}\text { Uncertain malignant } \\
\text { potential }\end{array}$ & $75, \mathrm{~F}$ & 1 & 3 & No & Yes & Intact & 1 & HNF1- $\alpha$ inactivated \\
\hline 23 & $\begin{array}{l}\text { Uncertain malignant } \\
\text { potential }\end{array}$ & $49, \mathrm{~F}$ & 1 & 2.2 & No & Yes & Intact & 2 & HNF1- $\alpha$ inactivated \\
\hline 24 & $\begin{array}{l}\text { Uncertain malignant } \\
\text { potential }\end{array}$ & $45, \mathrm{M}$ & 1 & 3.3 & No & Yes & Intact & 1 & HNF1- $\alpha$ inactivated \\
\hline 25 & $\begin{array}{l}\text { Uncertain malignant } \\
\text { potential }\end{array}$ & $46, \mathrm{~F}$ & $\begin{array}{l}4 \text { (including } 2 \text { non- } \\
\text { pigmented adenomas) }\end{array}$ & 13 & No & Yes & Intact & 3 & HNF1- $\alpha$ inactivated \\
\hline 26 & $\begin{array}{l}\text { Uncertain malignant } \\
\text { potential }\end{array}$ & $49, \mathrm{~F}$ & $\begin{array}{l}7 \text { (including } 4 \text { non- } \\
\text { pigmented adenomas) }\end{array}$ & $\begin{array}{l}\text { Unavailable, biopsy } \\
\text { specimen }\end{array}$ & Yes & No & Focal disruption & 2 & $\beta$-catenin activated \\
\hline 27 & $\begin{array}{l}\text { Uncertain malignant } \\
\text { potential }\end{array}$ & $25, \mathrm{M}$ & 2 & $\begin{array}{l}\text { Unavailable, biopsy } \\
\text { specimen }\end{array}$ & No & Yes & Intact & 2 & Inflammatory \\
\hline
\end{tabular}

Abbreviations: F, female; M, male.

a If multiple lesions are present, the recorded size is that of the largest lesion.

$\mathrm{b}$ If multiple lesions are present, the recorded score is that of the most pigmented lesion. 

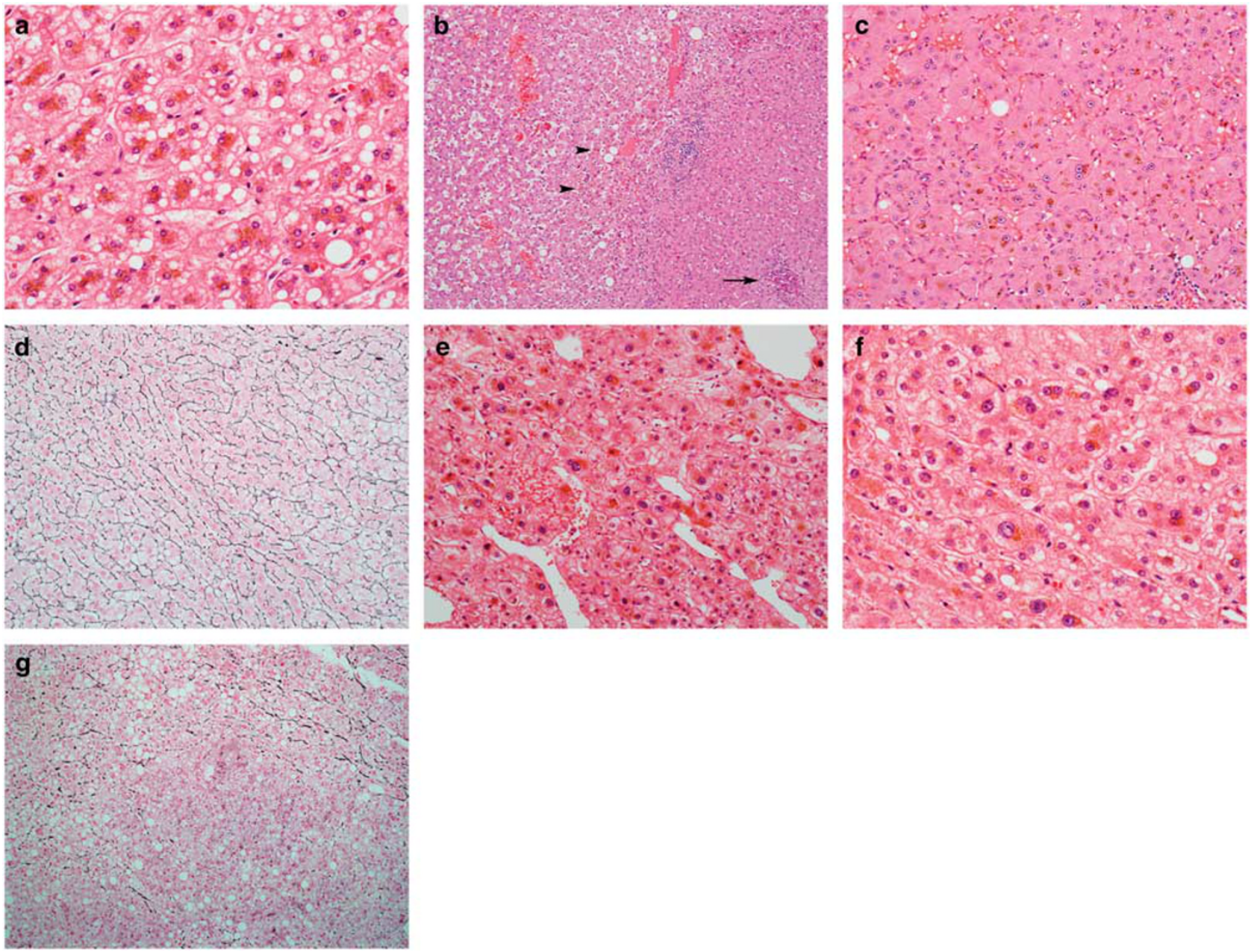

Figure 1 (a and b) Pigmented hepatocellular adenoma. This pigmented hepatocellular neoplasm shows no cytological or architectural atypia. Mild fatty change is present (a, $400 \times)$. (b) $(100 \times)$ Another example of an inflammatory-type hepatocellular adenoma with patchy pigmentation (arrowheads), inflammation, sinusoidal dilatation, and an abortive portal tract (structure composed of arterial branches invested in connective tissue and mild inflammation, but lacking a native bile duct. It is typically seen in inflammatory-type hepatocellular adenoma and is indicated here by an arrow) are evident. Reticulin stain showed an overall intact reticulin meshwork in all pigmented hepatocellular adenomas (not shown). (c and d) Pigmented hepatocellular neoplasm of uncertain malignant potential: this pigmented hepatocellular neoplasm shows evident cytological atypia but no significant architectural abnormalities by H\&E (c, $200 \times$ ) and has an overall intact reticulin meshwork (d: $100 \times$ ); the atypia falls short of a diagnosis of hepatocellular carcinoma, and a diagnosis of hepatocellular neoplasm of uncertain malignant potential was rendered. (e-g) Pigmented hepatocellular carcinoma. this pigmented hepatocellular neoplasm demonstrates thick trabecular growth $(\mathbf{e}, 200 \times)$ and marked cytological atypia $(\mathbf{e}, 200 \times$ and $\mathbf{f}, 400 \times)$. A reticulin stain $(\mathrm{g}, 40 \times)$ shows marked reduction of reticulin within the lesion; the H\&E and reticulin findings are consistent with hepatocellular carcinoma.

adenomas. The pigment score was 1 in $44 \%$ of cases, 2 in $30 \%$ of cases, and 3 in $26 \%$ of cases. The pigment intensity score was 1 in 6 hepatocellular adenomas, 2 hepatocellular neoplasms of uncertain malignant potential, and 4 hepatocellular carcinoma. The score was 2 in 2 hepatocellular adenomas, 4 hepatocellular neoplasms of uncertain malignant potential, and 2 hepatocellular carcinomas. The score was 3 in 3 hepatocellular adenomas, 1 hepatocellular neoplasm of uncertain malignant potential, and 3 hepatocellular carcinomas (Figure 3). The intensity of the pigment did not correlate with patient gender, age, tumor size, atypia, malignancy, or tumor phenotype. All cases were negative for iron.

The intracytoplasmic pigment showed intense granular staining with glypican-3 in 24 (89\%) cases
(Figure 4), but no diffuse cytoplasmic staining was observed. Ki-67 was very low $(<1 \%)$ in $25(93 \%)$ cases, and increased in 2 cases $(2 \%$ in 1 hepatocellular neoplasm of uncertain malignant potential and $7 \%$ in 1 hepatocellular carcinoma) (Figure 4). Liver fatty acid-binding protein was lost in 14 of 27 cases, 9 cases had nuclear $\beta$-catenin or diffuse glutamine synthetase expression, and 5 cases were positive for C-reactive protein or serum amyloid-A (Figure 4). The overall phenotype was consistent with isolated HNF-1 $\alpha$ inactivation in 13 tumors (48\%), which included 8 hepatocellular adenomas, 4 hepatocellular neoplasms of uncertain malignant potential, and 1 hepatocellular carcinoma. Isolated $\beta$-catenin activation was seen in 7 cases $(26 \%)$, including 1 hepatocellular neoplasm of uncertain malignant 

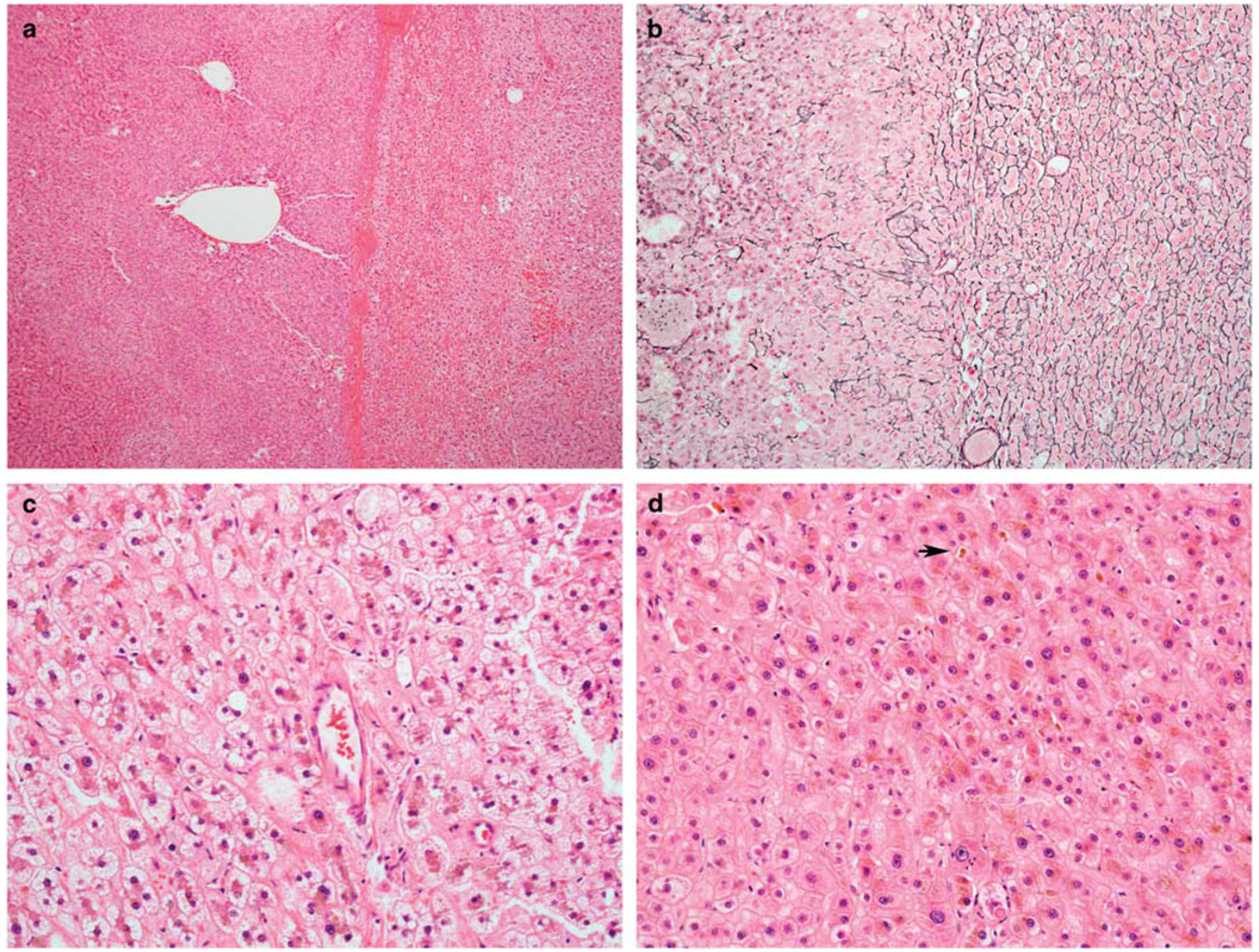

Figure 2 Hepatocellular carcinoma arising in hepatocellular adenoma. All cases of hepatocellular carcinoma arising in hepatocellular adenoma showed a 'nodule in nodule' growth (a, hepatocellular adenoma on the right and hepatocellular carcinoma on the left side, $40 \times$ ). A reticulin stain $(\mathbf{b}, 40 \times)$ of this same area shows reticulin loss in the hepatocellular carcinoma component (b, left) and reticulin retention in the hepatocellular adenoma component (b, right). Although atypia is absent in the hepatocellular adenoma component $(\mathbf{c}, 200 \times)$, the hepatocellular carcinoma component shows evident atypia (D, 200 × ) and cholestasis (d, arrow).

potential, and 6 hepatocellular carcinomas. An isolated inflammatory phenotype was seen in 4 cases (15\%), including 1 hepatocellular adenoma, 2 hepatocellular neoplasms of uncertain malignant potential, and 1 hepatocellular carcinoma. Concurrent $\beta$-catenin activation and inflammatory phenotype was seen in 1 hepatocellular adenoma and concurrent HNF-1 $\alpha$ inactivation and $\beta$-catenin activation was seen in another single hepatocellular adenoma. One carcinoma remained unclassified.

By electron microscopy, the pigmented material present within neoplastic hepatocytes had typical ultrastructural characteristics of lipofuscin, featuring polymorphic, electron dense, and granular material that is delimited within lysosomes (Figure 5).

Of 19 lesions with available background liver for review, $89 \%$ had easily visible lipofuscin in the background liver. The grade of lipofuscin pigment deposition in the background liver of pigmented tumors showed an average score of $1.3 \pm 0.8$ (range of
0-2), and was significantly higher than that of the control group, which had an average score of $0.3 \pm 0.6$ (range of $0-2)(P=0.0012)$.

\section{Discussion}

This study describes the morphological and genotype-phenotype characteristics of well-differentiated hepatocellular tumors with pigment and evaluates their association with atypia and malignancy. On the basis of our findings, pigment can be found in $\sim 25 \%$ of cases. In most cases, this pigment is light and can be overlooked when focusing on other aspects of making a diagnosis. However, cases with very prominent pigment accumulation (score of 3) were uncommon, accounting for only $6.4 \%$ of all cases. It is this latter category that is typically referred to as pigmented adenomas in the literature. ${ }^{10-16}$ Because we noticed that pigmentation in hepatocellular 

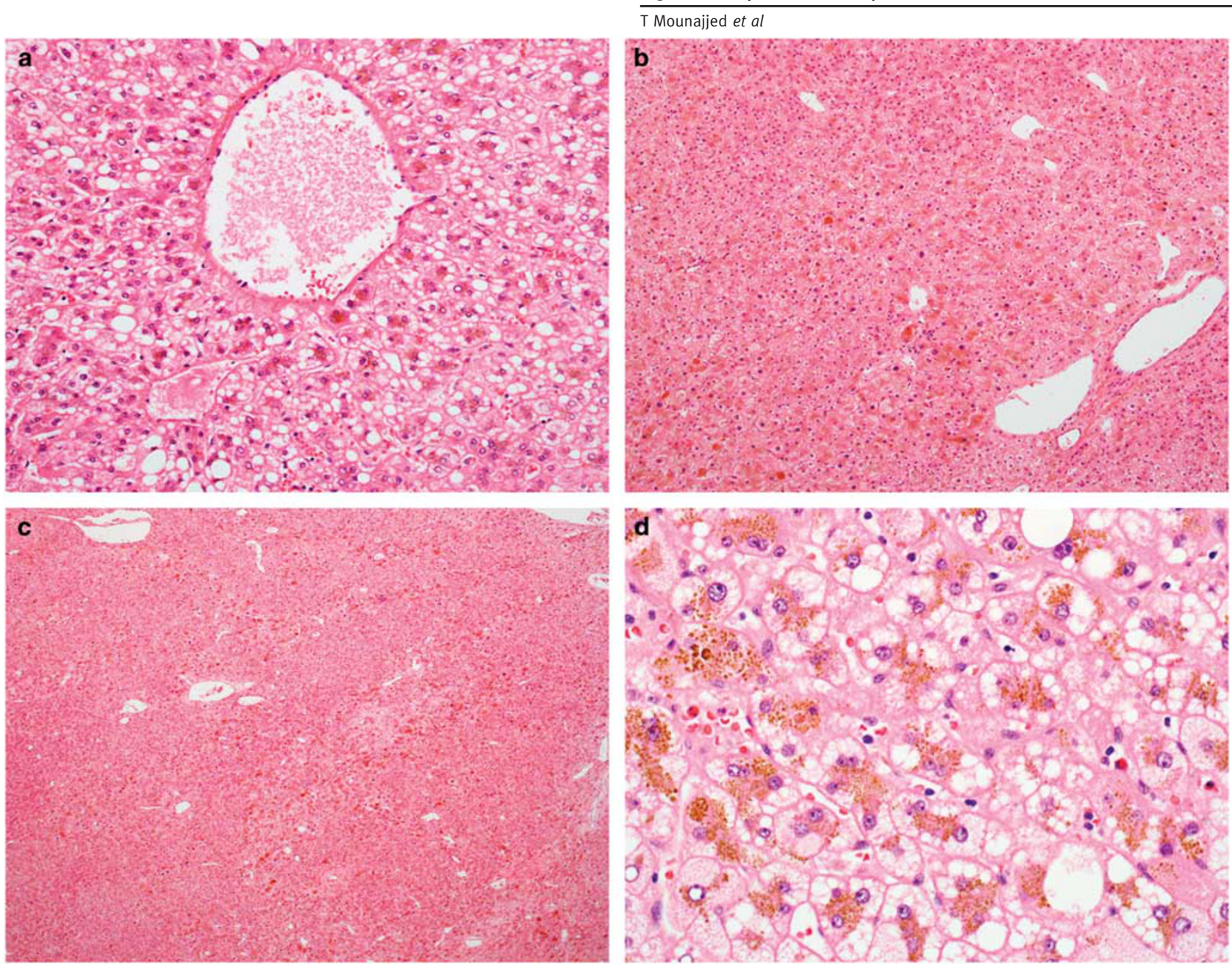

Figure 3 Semiquantitative scoring of pigment deposition in pigmented hepatocellular neoplasms. (a) Score 1+: pigment deposition is focal, and is seen in a minority of $100 \times$ fields. (b) Score 2+: the pigment is identified easily at $100 \times$ magnification (majority of $100 \times$ fields). (c) Score 3+: the pigment is easily identified at low power $(40 \times)$ magnification. (d) Pigment appearance: high power $(400 \times)$ examination of the pigment in pigmented hepatocellular neoplasms shows a granular brownish pigment deposited within neoplastic hepatocytes.

neoplasms has a spectrum of intensity and distribution, and because of the lack of a clear delineation of what constitutes a 'heavily pigmented adenoma' in previous studies, we decided to include any lesion containing 'easily identifiable pigment' in this series, ie, lesions with pigment visible at $100 \times$ magnification. Hence, the latter criterion was used in this study as a reproducible feature in all pigmented hepatocellular neoplasms. On the basis of our findings, the intensity of the pigment did not correlate with any specific clinicopathological features. In contrast to the small group of heavily pigmented lesions (3+), all pigmented lesions (1+ to $3+$ ) constituted a distinct entity characterized by increased risk of atypia and malignancy and association with increased lipofuscin in the background liver. For these reasons, we propose including neoplasms with any amount of pigment that can be identifiable at $100 \times$ magnification in the "pigmented' category.

The immunophenotype of pigmented adenomas has not been systematically investigated but isolated reports describe both the inflammatory phenotype and $\beta$-catenin activated phenotype. ${ }^{10,15}$ The findings in this study demonstrate the immunophenotype in pigmented adenomas is heterogeneous. HNF- $1 \alpha$ inactivation is the most common phenotype overall, and is the most common phenotype in the subgroups of hepatocellular adenomas without atypia and hepatocellular adenomas with atypia (hepatocellular neoplasm of uncertain malignant potential). In contrast, $\beta$-catenin activation was the most common phenotype in cases classified as hepatocellular carcinoma. Interestingly, two hepatocellular adenomas had somewhat less common phenotypes, one with concurrent $\beta$-catenin activation and inflammatory phenotype, and one with concurrent HNF-1 $\alpha$ inactivation and $\beta$-catenin activation. Rare cases with similar mixed phenotypes have also been reported previously. ${ }^{19}$

On the basis of our findings, pigmented tumors have a high association with atypia and malignancy. Overall, hepatocellular carcinoma or some degree of histological atypia was present in $59 \%$ of cases. 

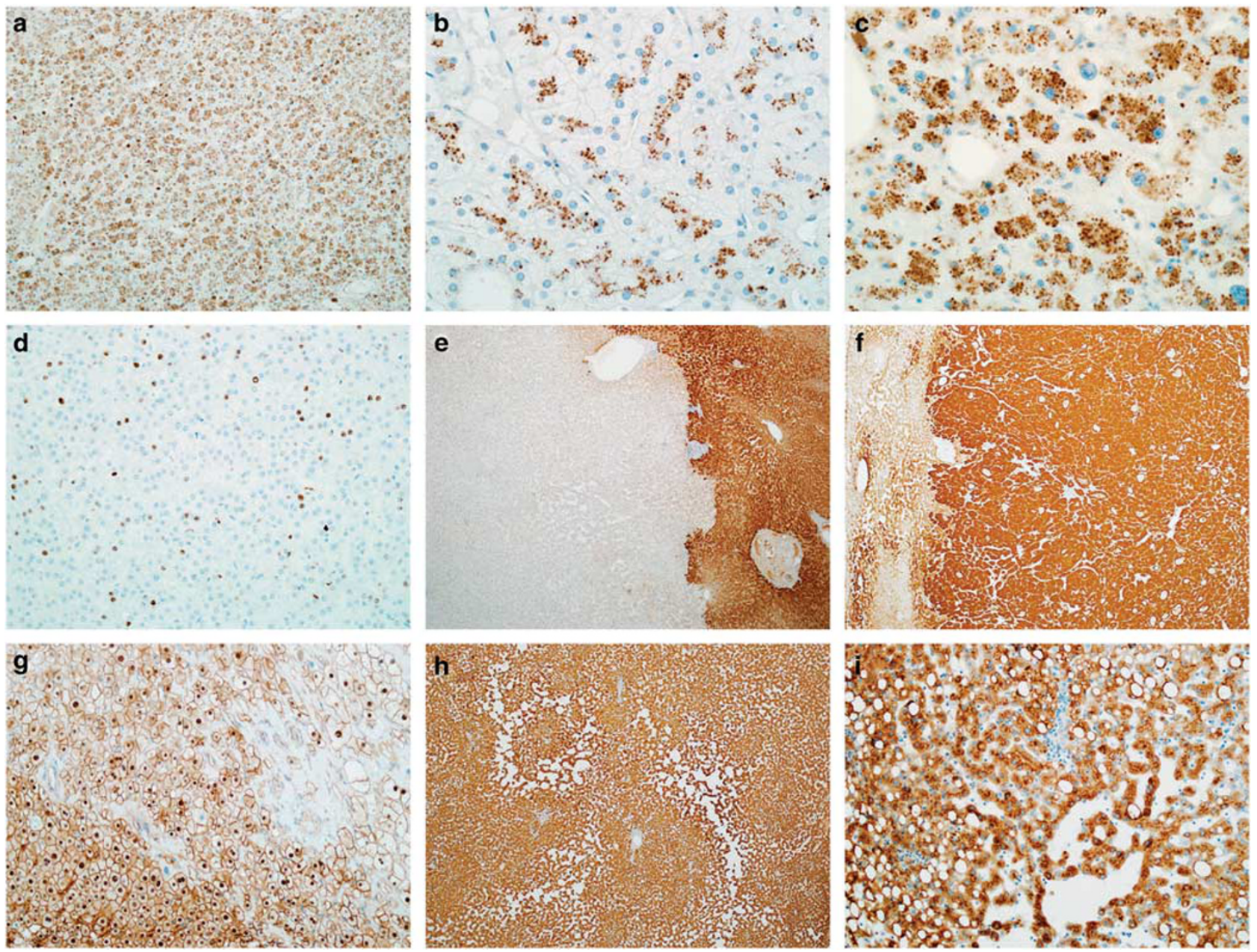

Figure 4 Immunohistochemical features of pigmented hepatocellular neoplasms. (a-c) Glypican-3: the pigment within tumor cells is highlighted by glypican-3 immunostain $(\mathbf{a}, 100 \times)$. The staining pattern can be pericanalicular $(\mathbf{b}, 400 \times)$ or more diffuse $(\mathbf{c}, 400 \times)$ in cases with heavy pigment deposition. (d): Ki-67 $(400 \times)$ : this is the only example of a hepatocellular carcinoma showing increased proliferative index by Ki-67. (e): Liver fatty acid-binding protein $(40 \times)$ : this example of a HNF-1 $\alpha$ inactivated hepatocellular adenoma shows loss of liver fatty acid-binding protein expression in the tumor (left) compared with the non-neoplastic liver (right). (f and g): Glutamine synthetase and $\beta$-catenin: this example of a $\beta$-catenin activated hepatocellular neoplasm of uncertain malignant potential shows diffuse expression of glutamine synthetase (f, $40 \times$ ) in the tumor (f, right) in comparison with localized perivenular staining in the non-neoplastic liver (f, left). Aberrant nuclear expression of $\beta$-catenin was also present (g, $200 \times$ ). (h and i) C-reactive protein and serum amyloid-A: this example of inflammatory-type hepatocellular adenoma shows diffuse strong expression of both C-reactive protein (h, $40 \times)$ and serum amyloid-A (i, $200 \times)$.

Furthermore, in individuals with multiple hepatocellular neoplasms, it was only the pigmented lesions that showed changes of hepatocellular neoplasm of uncertain malignant potential or hepatocellular carcinoma. Malignant transformation of pigmented hepatocellular adenomas was also relatively common. These findings indicate that pigmented hepatocellular neoplasms have an increased risk of malignancy. This risk was even more striking in men, as all tumors in men were at least atypical and $67 \%$ were classified as malignant. This risk in men is unlikely to be entirely explained by the presence of pigment, as men with hepatocellular adenomas have a generalized increase risk of malignancy, which in some studies exceeds 10 times that of women. ${ }^{20,21}$ Our findings, however, suggest that this risk is even higher in males with pigmented neoplasms.

Electron microscopy findings show the pigmented material is lipofuscin. Although a few cases had spatial or specific intracellular localization of pigment (perivascular or pericanalicular), in most cases the pigment deposition was random (azonal). Glypican-3 is strongly positive in the lipofuscin material, highlighting an important potential diagnostic pitfall; this staining pattern of lipofuscin should be distinguished from the diffuse cytoplasmic staining of hepatocellular carcinoma.

The background livers in cases of pigmented tumors also contain significantly higher lipofuscin content, when compared with livers containing non-pigmented hepatocellular adenomas. This observation raises the 

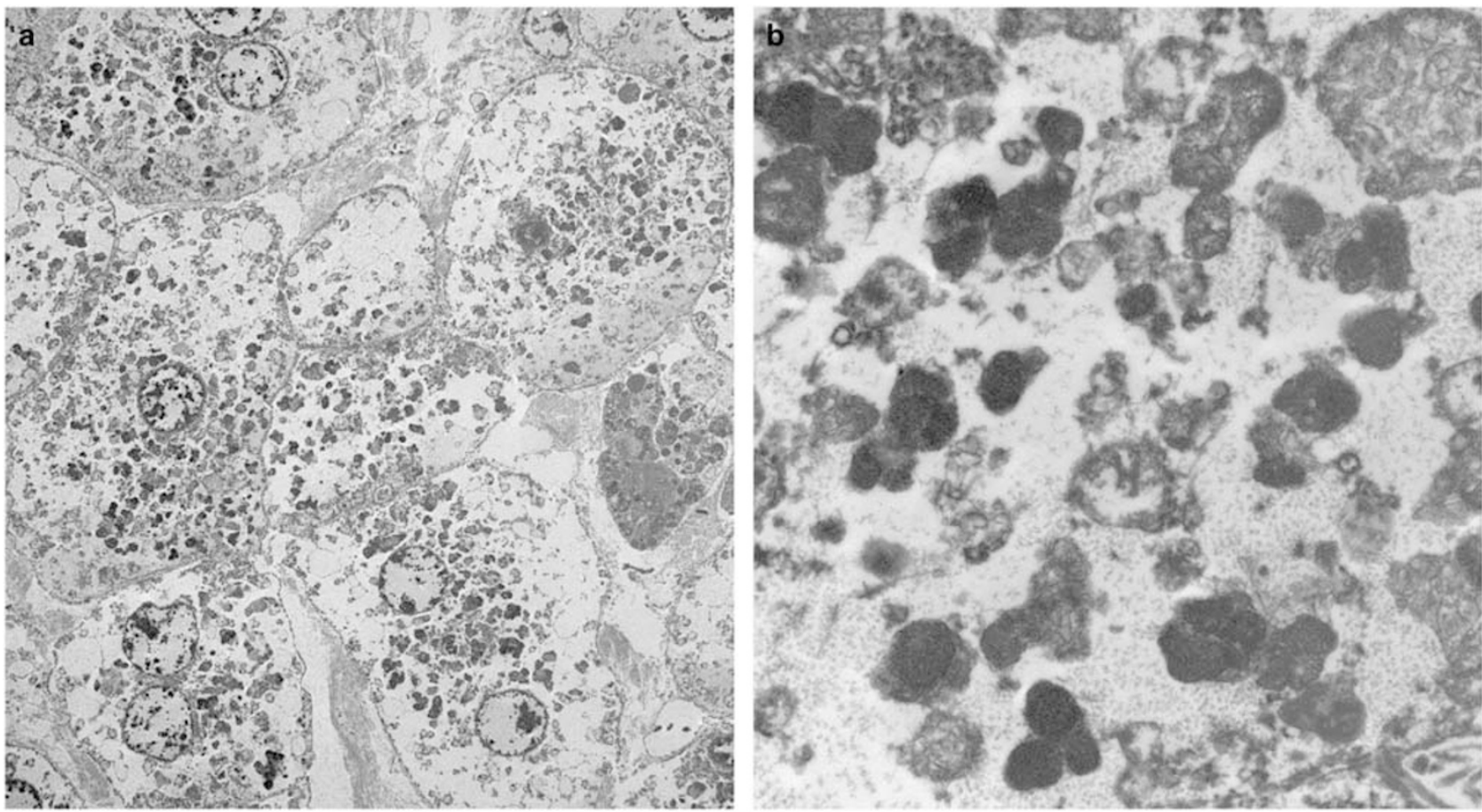

Figure 5 Electron microscopic features of pigment in pigmented hepatocellular neoplasms. The pigmented material present within neoplastic hepatocytes has ultrastructural features typical of lipofuscin, characterized by polymorphic, electron dense, and granular material that is delimited within lysosomes.

possibility that lipofuscin accumulation in hepatocellular neoplasms may reflect genetic changes or environmental influences relevant to the entire liver. Of note, however, not all hepatocellular neoplasms arising in the livers with background lipofuscin were pigmented, but only the pigmented neoplasms were associated with atypia and malignancy. This suggests that lipofuscin accumulation in hepatocellular adenomas, regardless of its cause, serves as a marker for increased risk of progression towards malignancy.

Lipofuscin consists of highly oxidized lipids that accumulate in lysosomes as a result of cell aging and damage. Although lipofuscin occurs frequently in benign cells, it has been linked indirectly to carcinogenesis by its association with cell senescence and increased peroxisomal oxidation. ${ }^{22-24}$ Both of these cell conditions, wherein lipofuscin is a morphological correlate, can result in carcinogenesis through oxidative damage to the DNA. For instance, carcinogenesis often arises in senescent cells that are still competent for replication. ${ }^{23}$ Furthermore, in animal models, peroxisome proliferator-induced hepatocellular carcinogenic activity was associated with abundant lipofuscin accumulation in the background liver. ${ }^{24}$ Although our findings suggest that lipofuscin accumulation in neoplastic hepatocytes is associated with atypia and malignancy, its exact pathogenetic role remains uncertain, but may be related to its role as an indicator of DNA damage through oxidation.

In summary, our data indicate that any degree of lipofuscin pigment is seen in up to $25 \%$ of hepatocellular adenomas, with $6 \%$ of cases showing heavy pigmentation (3+ staining), the latter group traditionally referred to as pigmented adenomas. Phenotypically, pigmented adenomas represent a heterogeneous group of tumors, with HNF-1 $\alpha$ inactivation being the commonest phenotype. By electron microscopy, the pigmented material is most consistent with lipofuscin, whose accumulation in the lesions may be a byproduct of generalized increase in hepatic lipofuscin. Nevertheless, our data indicate that these lipofuscin-containing lesions have an increased risk of atypia and malignancy, with most classified as hepatocellular neoplasm of uncertain malignant potential or hepatocellular carcinoma. Male gender is especially associated with malignancy. Further studies are needed to understand what mechanisms place these lipofuscin-containing lesions at an increased risk of atypia and malignancy.

\section{Disclosure/conflict of interest}

The authors declare no conflict of interest.

\section{References}

1 Rooks JB, Ory HW, Ishak KG et al. Epidemiology of hepatocellular adenoma. The role of oral contraceptive use. JAMA 1979;242:644-648.

2 Mays ET, Christopherson W. Hepatic tumors induced by sex steroids. Semin Liver Dis 1984;4:147-157. 
3 Bioulac-Sage P, Blanc JF, Rebouissou S et al. Genotype phenotype classification of hepatocellular adenoma. World J Gastroenterol 2007;13:2649-2654.

4 Bioulac-Sage P, Rebouissou S, Thomas C et al. Hepatocellular adenoma subtype classification using molecular markers and immunohistochemistry. Hepatology 2007;46:740-748.

5 Bioulac-Sage P, Balabaud C, Zucman-Rossi J. Subtype classification of hepatocellular adenoma. Dig Surg 2010;27:39-45.

6 Zucman-Rossi J, Jeannot E, Nhieu JT et al. Genotypephenotype correlation in hepatocellular adenoma: new classification and relationship with HCC. Hepatology 2006;43:515-524.

7 van Aalten SM, Verheij J, Terkivatan T et al. Validation of a liver adenoma classification system in a tertiary referral centre: implications for clinical practice. J Hepatol 2011;55:120-125.

8 Rebouissou S, Bioulac-Sage P, Zucman-Rossi J. Molecular pathogenesis of focal nodular hyperplasia and hepatocellular adenoma. J Hepatol 2008;48: 163-170.

9 Bioulac-Sage P, Balabaud C, Wanless IFocal nodular hyperplasia and hepatocellular adenomaIn:Bosman FTWHO Classification of Tumours of the Digestive System4th ednInternational Agency for Research on Cancer: Lyon, FranceLyon, France2010, pp 198-204.

10 Hechtman JF, Raoufi M, Fiel MI et al. Hepatocellular carcinoma arising in a pigmented telangiectatic adenoma with nuclear beta-catenin and glutamine synthetase positivity: case report and review of the literature. Am J Surg Pathol 2011;35:927-932.

11 Masuda T, Beppu T, Ikeda K et al. Pigmented hepatocellular adenoma: report of a case. Surg Today 2011;41: 881-883.

12 Koea JB, Kua H. Pigmented liver cell adenoma: rare but benign. ANZ J Surg 2012;82:86-87.

13 Hasan N, Coutts M, Portmann B. Pigmented liver cell adenoma in two male patients. Am J Surg Pathol 2000;24:1429-1432.

14 Vij M, Patra S, Rela M. Pigmented hepatocellular adenoma with complete CD34 immunostaining pattern: a diagnostic dilemma. Indian J Pathol Microbiol 2012;55:528-530.

15 Kim GJ, Seok JY, Rhee H et al. beta-Catenin activated hepatocellular adenoma: a report of three cases in Korea. Gut Liver 2014;8:452-458.

16 Roth JA, Berman E, Befeler D et al. A black hepatocellular carcinoma with Dubin-Johnson-like pigment and Mallory bodies: a histochemical and ultrastructural study. Am J Surg Pathol 1982;6:375-382.

17 Craig JR, Peters RL, Edmondson HA.Tumors of the liver and intrahepatic bile ducts. Atlas of Tumor Pathology. Armed Forces Institute of Pathology: Washington DC, USA 1989

18 Bedossa P, Burt AD, Brunt EM et al. Well-differentiated hepatocellular neoplasm of uncertain malignant potential: proposal for a new diagnostic category. Hum Pathol 2014;45:658-660.

19 Kim H, Jang JJ, Kim DS et al. Clinicopathological analysis of hepatocellular adenoma according to new bordeaux classification: report of eight korean cases. Korean J Pathol 2013;47:411-417.

20 Farges O, Ferreira N, Dokmak S et al. Changing trends in malignant transformation of hepatocellular adenoma. Gut 2011;60:85-89.

21 Dokmak S, Paradis V, Vilgrain V et al. A single-center surgical experience of 122 patients with single and multiple hepatocellular adenomas. Gastroenterology 2009;137:1698-1705.

22 Marsman DS, Goldsworthy TL, Popp JA. Contrasting hepatocytic peroxisome proliferation, lipofuscin accumulation and cell turnover for the hepatocarcinogens Wy-14,643 and clofibric acid. Carcinogenesis 1992;13: 1011-1017.

23 Gosselin K, Martien S, Pourtier A et al. Senescenceassociated oxidative DNA damage promotes the generation of neoplastic cells. Cancer Res 2009;69: 7917-7925.

24 Reddy JK, Lalwani ND, Reddy MK, Qureshi SA. Excessive accumulation of autofluorescent lipofuscin in the liver during hepatocarcinogenesis by methyl clofenapate and other hypolipidemic peroxisome proliferators. Cancer Res 1982;42:259-266. 